\title{
1 Equine meniscal degeneration is associated with medial femorotibial osteoarthritis
}

2 Keywords: equine; meniscus; osteoarthritis; femorotibial

3 Word count: 4969

4

5 SUMMARY

6

7 Background: There is limited information available concerning normal equine meniscal

8 morphology, its degeneration and role in osteoarthritis (OA).

9 Objectives: To characterize normal equine meniscal morphology and lesions and to explore the

10 relationship between equine meniscal degeneration and femorotibial OA.

11 Study Design: Ex vivo cadaveric study.

12 Methods: Menisci were harvested from 7 normal joints $(n=14$ menisci) and 15 joints with OA (n

$13=30$ menisci). A macroscopic femorotibial OA score (cartilage degeneration and osteophytosis)

14 was employed to measure disease severity in each compartment. The femoral and tibial meniscal

15 surfaces were scored for macroscopic fibrillation and tears (1-4). Histological sections (Regions:

16 cranial and caudal horn; body) were also scored for microscopic fibrillation and tears (0-3) and

17 inner border degeneration (0-3).

18 Results: Partial meniscal tears were present on both femoral and tibial surfaces in all 3 regions and

19 most frequently identified on the femoral surface of the cranial horn of the medial meniscus and

20 body of the lateral meniscus. There was a significantly positive correlation between the global

21 medial meniscal macroscopic scores and osteophyte $(\mathrm{r}=0.7, \mathrm{p}=0.002)$ or cartilage degeneration

$22(\mathrm{r}=0.5, \mathrm{p}=0.03)$ scores within the medial femorotibial joint. The global medial meniscal

23 macroscopic score was greater $(\mathrm{p}=0.004)$ in the advanced OA joints compared with control joints. 
24 Main limitations: The menisci were principally from abattoir specimens without a known clinical

25 history because of the challenge in obtaining a large number of specimens with a clinical diagnosis

26 of femorotibial OA.

27 Conclusions: This study is the first to describe normal equine meniscal morphology and lesions.

28 Meniscal lesions were identified in all segments and on both articular surfaces. Meniscal

29 degeneration significantly correlated with OA severity in the equine medial femorotibial joint. The

30 relationship between OA and meniscal pathology remains to be elucidated and we speculate that

31 mechano-biological events play a role. 
Stifle lameness accounted for $42 \%$ of hindlimb referral lameness in eventing horses[1].

34 However, this data does not represent all equine athletic disciplines. For example, in racehorses, it

35 is probably much lower, but the exact prevalence is not known. Meniscal injury has been reported to account for up to $20 \%$ of all stifle lamenesses [2; 3]. We also recently reported that OA pathology

37 is most frequently observed in the medial femorotibial compartment of the stifle[4]. Equine

38 meniscal lesions have been identified in the cranial horn of the meniscus on arthroscopic

39 examination [3;5-9] and in all 3 meniscal segments on ultrasonographic examination $[3 ; 6-11]$.

40 However, only one third of the femoral surface of the meniscus is visible by arthroscopy $[5 ; 12]$.

41 Macroscopic lesions of the remaining meniscal femoral or tibial surfaces and intrasubstance

42 degenerative lesions are not visualized on arthroscopic examination [7]. Approximately half

$43(13 / 25)$ of the lesions diagnosed on ultrasonographic examination of equine menisci were not

44 observed on arthroscopic examination [7] thus underpinning the importance of a meniscal

45 ultrasonographic examination. However ultrasonographic visualization of the caudal menisci is

46 limited [9]. Open, large bore magnetic resonance imaging (MRI) technology, accommodating all

47 sizes of equine stifles [13], holds the promise to provide a more accurate diagnosis and improve 48 understanding of equine meniscal disease as MRI is the gold standard for evaluation of meniscal

49 disease in man [14].

50 The menisci are semilunar structures that enhance the congruency between the femoral

51 condyles and tibial plateau, transfer load and are paramount for femorotibial joint stability $[6 ; 15]$.

52 Meniscal tears have been classified in man and include longitudinal (or bucket-handle when they

53 penetrate deeper into the meniscal substance), vertical, horizontal, radial, oblique and complex

54 tears [16]. The incidence of meniscal tears identified on MRI in a study of the relationship of 55 meniscal disease and OA in man was $16 \%$ in control patients and increased to $57 \%$ in OA patients 
56 [17]. Furthermore, loss of meniscal intra-substance integrity has recently been shown to be

57 correlate with risk factors for cartilage degeneration in man [18]. Knowledge of equine meniscal

58 morphology and lesion types is particularly important to avoid interpretation of normal structural

59 artefacts on ultrasonographic examination leading to a misdiagnosis [7].

60 We postulate that equine meniscal and articular cartilage degeneration are interlinked by

61 mechanobiological events. The objectives of the present investigation were: 1 ) to characterize

62 normal equine meniscal morphology and lesions and 2) to explore the association between

63 meniscal degeneration, aging and equine OA.

64 


\section{Specimens}

68 The study protocol was approved by the institutional Animal Care and Use Committee.

69 Menisci investigated (Table 1) were from the stifle joints of adult horses $(n=21)$, characterized in

70 a previous imaging investigation of stifle $\mathrm{OA}[4]$ and stored in a tissue bank. Additional menisci

71 from donated horses were included: $(n=2)$. One joint was included per animal. The joints were

72 either placed in saline soaked gauze and frozen at $-20^{\circ} \mathrm{C}$ or processed immediately following 73 euthanasia.

\section{Macroscopic assessment}

Menisci were thawed in water. The macroscopic changes were scored by consensual agreement of 2 individuals, blinded to the pathology (articular cartilage degeneration and osteophytes) previously assessed in that joint compartment [4]. Exceptionally, 1 pair of menisci was evaluated by only one individual, at the end of the study. The macroscopic changes (fibrillation and tears) in each of the 3 regions (cranial horn; body and caudal horn, Figure 1) of either the tibial and femoral meniscal surfaces were scored (details in Figure 2; modified from Pauli et al.[19])

82 following the application of India Ink. The tibial or femoral surface macroscopic meniscal score

83 was the cumulative scores of the three regions, whereas the global meniscal macroscopic score was

84 the sum of the scores on both surfaces. 
The menisci were placed in 10\% formaldehyde for 2 hours and transferred to EDTA $20 \%$

89 for 2 weeks, to facilitate sectioning. Each meniscus was then laid over a protractor with the femoral

90 surface uppermost and the cranial border aligned with the angle 0 . Three slices $(\approx 0.5 \mathrm{~cm}$ thick $)$

91 were cut at 30,90 and $150^{\circ}$ (Figure 1) and embedded in paraffin. Five $\mu \mathrm{m}$ sections were cut and

92 stained with HEPS (hematoxylin, eosin, phloxine and saffron). All slides were digitalized with a

93 LeicaDM 4000B microscope and Panoptiq ${ }^{\mathrm{TM}}$ v.1.4.3 computer software.

HEPS stained sections from macroscopically normal appearing menisci of selected horses

96 of different ages were first examined to describe normal meniscal histological features at different

97 ages. The histologic lesions (fibrillation, disruption, lack of tissue) were scored (details in Figure

98 2; modified from Pauli et al.[19]). Each section was graded independently by 2 observers, one a

99 board certified pathologist. Histologic changes on both the femoral and tibial surfaces and inner

100 border were assessed. The tibial or femoral surface histologic meniscal score was the sum of this

101 parameter score and inner border score from the 3 regions, whereas the global meniscal histologic

102 score was the cumulative scores recorded in the 3 sections.

103

meniscal scores between the lateral and medial menisci. A Wilcoxon test was used to detect differences between the regional macroscopic meniscal scores and total tibial or femoral surface macroscopic scores between the medial and lateral menisci. The same test was used for the global

108 histologic meniscal score comparison between the lateral and medial menisci. The regional 109 macroscopic and histological meniscal scores within the medial or lateral menisci were assessed to 
110 identify differences with a Friedman test for non-parametric values and then with Tukey post-hoc

111 tests when needed.

112

\section{Assessment of meniscal lesion association with femorotibial compartment OA}

114 In order to assess the association between meniscal degeneration and OA, global

115 femorotibial compartment macroscopic OA scores, calculated in a prior recently published study

116 [4] were employed. Specimens where the femorotibial joint OA had been scored were included for

117 this arm of the study (Table 1). Briefly, the articular cartilage changes (fibrillation and erosion; 0-

1183 ) and osteophytes (0-3) were scored in the cranial, middle and caudal regions of the femoral

119 condyles and tibia. The regional scores were summed to provide a total femorotibial compartment

120 macroscopic cartilage (0-9) or osteophyte score (0-9) or a global femorotibial compartment

121 macroscopic OA score (all cartilage and osteophyte scores summed) (Table 1). The menisci were

122 also categorized into arbitrary groups, based on their corresponding global femorotibial

123 compartment macroscopic OA score [4]: control (a score of $<5$; no osteophytes); moderate OA $(\geq$

1245 to $<20)$ and advanced OA (a score of $\geq 20)$ to further elucidate the association between meniscal

125 degeneration and OA lesions.

126 A Spearman correlation coefficient was employed to correlate the global medial and lateral

127 meniscal macroscopic and histological scores with the total femorotibial compartment macroscopic

128 osteophyte and cartilage scores to identify correlations between meniscal lesion scores and OA in

129 each femorotibial compartment. Kruskal-Wallis tests were employed to detect differences between

130 global medial and lateral meniscal macroscopic and histologic scores in groups categorized by OA

131 status (control, OA moderate, or advanced OA). Post-hoc tests[20] were performed on the

132 statistically significant findings to reveal the direction of the differences. 


\section{Association of meniscal degeneration with age}

Meniscal specimens with a known age were assessed (specimens 2-7, 11, 12, 14-17, 19,

136 21-23, $n=32$ menisci, Table 1). A mixed ANCOVA was employed on the global macroscopic and

137 histologic meniscal scores with age as co-factor, laterality as fixed factor and horse ID as random

138 factor to determine the association of meniscal degeneration and age. $A$ value $p=0.05$ was

139 considered significant.

\section{$141 \quad$ RESULTS}

\section{Normal menisci}

143 Histologic appearance: The menisci were wedge-shaped in cross-section, with a concave femoral

144 surface, a flat tibial surface and a superficial lamellar layer, which stained slightly orange with

145 HEPS (Figures $1 \& 2$ ). The central part of the meniscus was eosinophilic and the inner third was

146 also occasionally lightly eosinophilic (Figure 2). Matrix fibres were oriented radially at the femoral

147 and tibial meniscal surfaces. In each sample, the lamellar layer was subjectively more cellular and

148 cells were spindle-shaped in appearance compared to the cells of the central zone, that had a

149 heterogenous orientation (Figure 2). The cells of the inner border were round.

150 


\section{Meniscal lesions: location and frequency}

A landscape of macroscopic meniscal lesions was available for study spanning from mild

153 fibrillation of the surfaces or inner border, to tears and partial loss of tissue (Figure 3). Surface

154 fibrillation was often present alone, but also occurred in association with partial tears. The meniscal 155 tears were principally oriented longitudinally along the circumferential meniscal fibers (Figure 3

156 a) or obliquely on the femoral surface (Figure $3 \mathrm{~b} \& \mathrm{c}$ ). Macroscopic lesion scores and the 157 corresponding score percentage per region (cranial horn, body, caudal horn) of the femoral or tibial

158 surfaces are illustrated in Figure 4. The only score 4 meniscal lesion was found on a lateral

159 meniscus (Figure 4 a) and score 3 lesions (Figure $4 \mathrm{c}-\mathrm{d}$ ) were most prevalent in the cranial horn of

160 the medial meniscus and in the body of the lateral meniscus. On the tibial surface, score 3 was the

161 most severe lesion encountered and was most prevalent in the caudal horn of the medial meniscus

162 and in the body of the lateral meniscus (Figure 4).

164 Comparison of the global macroscopic meniscal scores and the tibial or femoral surface total

165 macroscopic scores between the medial and lateral menisci: Global macroscopic meniscal scores

166 (median, range) were higher $(10,6-14, \mathrm{p}=0.04)$ in the medial meniscus than those of the lateral

167 menisci $(8,6-16)$ (Figure 4 a). The tibial surface total macroscopic scores of the medial meniscus

168 were higher $(5,3-8, \mathrm{p}=0.02)$ compared with the lateral $(4,3-6)$, but no difference was detected for 169 the femoral surface scores.

171 Comparisons of regional macroscopic meniscal scores within the medial or lateral menisci: The

172 regional macroscopic scores (median, range) of the femoral and tibial surfaces of the medial

173 meniscus were higher in the meniscal body (femoral: 2, 1-3; tibial: 2, 1-3) compared to the caudal

174 (femoral: 1, 1-3; tibial: 2, 1-3; $\mathrm{p}=0.01$ ) and cranial horns (femoral: 2, 1-3; tibial: 1, 1-3; $\mathrm{p}=0.04$ ) 
175 respectively. No significant difference was detected between regional scores in the lateral 176 meniscus.

178 Histologic appearance: A variety of lesions were observed and spanned from surface changes and 179 undulation to complete meniscal tissue disruption. The majority of the lesions affected the lamellar 180 layer, with some penetration to the central zone. Representative lesions and corresponding scores 181 are provided in Figure 2. At the femoral surface, the highest median histologic score was 2 in the 182 cranial horn of the medial meniscus and 1 in the body of the medial meniscus on the tibial surface.

183 The highest median histologic score recorded at the inner border was 2 in both the body and caudal 184 horn of the medial meniscus.

Comparison of global histologic meniscal score and tibial, femoral surface or inner border total

187 histologic scores between the medial and lateral menisci: The global histologic medial meniscal 188 scores (median, range) were higher $(12,0-18, \mathrm{p}=0.01)$ compared with the lateral meniscus $(4,0$ 189 21) (Figure $4 \mathrm{~b}$ ). The inner border and tibial surface total histologic scores were higher $(\mathrm{p}=0.008$ 190 and 0.02 respectively) in the medial (inner border: 5, 0-9; tibial surface: $2,0-9$ ) compared to the 191 lateral meniscus (inner border: 1, 0-9; tibial surface: 1, 0-6) (Supplementary item 2). No other 192 differences were identified.

194 Comparisons of regional histologic meniscal scores within the medial or lateral menisci: The

195 medial meniscus regional femoral surface histologic score (median, range) was higher $(\mathrm{p}=0.003)$

196 in the cranial horn $(2,0-3)$ compared to the body $(0,0-2)$ and caudal horn $(0,0-2)$ and its regional

197 tibial surface histologic score was higher $(\mathrm{p}=0.009)$ in the body $(1,0-3)$ compared to its cranial 198 horn (0, 0-3). No other differences were detected. 
200 Association between meniscal lesion scores and OA in each femorotibial compartment

201 Significant correlations were identified in the medial femorotibial joint alone. Both global

202 medial meniscal macroscopic and histologic scores were positively correlated with the total

203 femorotibial compartment osteophyte scores $(r=0.7, p=0.002$ and $r=0.6, p=0.04$ respectively;

204 Figure $5 \mathrm{~b} \& \mathrm{~d}$ ). The global medial meniscal macroscopic score was also positively correlated with

205 the total femorotibial compartment macroscopic cartilage score $(r=0.5, p=0.03$; Figure 5 a).

206

207 Comparisons of meniscal pathology between OA groups

208 The global medial meniscal macroscopic and histologic scores (median, range) were greater

209 ( $\mathrm{p}=0.004$ and $\mathrm{p}=0.01$ respectively) in the advanced OA joints (macroscopic: $12,12-14$; histologic:

$21017,16-18$ ) compared to control joints (macroscopic 8, 6-9; histologic: 4, 2-12,). No other

211 significant associations were detected.

212

213 Meniscal degeneration and age

214 The global meniscal macroscopic and histologic scores increased with age $(\mathrm{p}<0.0001)$

215 (Figure 6). 
The findings of the present study provide valuable insight into equine meniscal disease.

218 First, a detailed description of macroscopic and histologic lesions of the equine meniscus is

219 provided. Second, we observed that meniscal macroscopic and histologic degeneration scores were 220 higher in the medial meniscus compared to its lateral counterpart, confirming previous clinical

221 reports $[3 ; 5-8 ; 10]$. Third, the medial meniscal macroscopic lesion scores were higher in its body 222 whereas the histologic scores were higher in the body of the meniscal tibial surface, but also in the

223 cranial horn on the femoral surface. Fourth, the meniscal degeneration scores correlated positively

224 with the macroscopic osteophyte and cartilage degeneration scores within the medial femorotibial 225 joint, suggesting a link to OA, similar to that observed in man [15].

226 The meniscal tears were oriented longitudinally in the direction of the circumferential meniscal fibres, or obliquely on the femoral surface. The macroscopic score 3 lesions we described are similar to longitudinal tears in man [16] whereas the score 4 lesions are comparable to complex tears [16].

The data presented here provides additional evidence that the equine medial meniscus is more frequently affected by pathology than its lateral counterpart $[3 ; 5-7 ; 10]$, similar to man [15].

232 These results confirm and extend those of Adrian et al. [7] who reported that more than half(25/47)

233 of the medial menisci examined ultrasonographically had lesions, compared to less than one fifth 234 (6/34) of the lateral menisci. However, no gold standard histopathological confirmation was 235 available in the latter study.

236 There are few studies on equine femorotibial or meniscal biomechanics published in the 237 English veterinary literature [21-24]. In contrast, this is a well studied area in man and it is known 238 that the medial meniscus withstands greater forces than its lateral counterpart and is the most 239 frequently injured [15]. The total axial forces generated in a human limb at a walk are at least 2 to 
2403 times body weight[25]. The knee joint transmits $65-73 \%$ of these forces with the remaining

241 transferred by surrounding soft tissues[26]. Furthermore, $85 \%$ of the peak force is transferred

242 through the medial side, depending on the valgus angle of the knee and this side can bear up to

$243201 \%$ of body weight at maximum axial load[26]. Although these findings cannot be directly

244 extrapolated to horses, the commonality of medial meniscal lesions in both suggests similarities in

245 etiology related to biomechanical loading events in the femorotibial joint compartment. Caution

246 should however be exercised when extrapolating the findings from human bipeds to equine

247 quadrupeds. To our knowledge, the forces transmitted through equine femorotibial joints in vivo

248 have been not been measured or reported in the English veterinary literature. Information from

249 quadruped dogs reveal that the fore limbs support $63 \%$ of body mass during standing and at all

250 walking speeds[27]. This information may also apply to the horse at similar gaits. The

251 commonality of medial meniscal lesions in humans, horses and dogs suggests similarities in

252 etiology related to biomechanical loading events in the femorotibial joint compartment, but

253 requires further study.

254 In addition to the laterality of meniscal lesions, we also analyzed regional site prevalence

255 of lesions and their severity. We observed that equine meniscal lesions arise in all 3 meniscal

256 segments in agreement with others [7], but also on both the femoral and tibial surfaces. When the

257 site prevalence of medial meniscal lesions was studied more closely, score 3 macroscopic lesions

258 were most commonly located in the cranial horn of the medial meniscus on the femoral surface.

259 This site prevalence may, in part, be explained by the results a recent equine meniscal

260 biomechanical study [22] that identified a caudal translocation of equine menisci occurring from

261 full extension to full flexion of the stifle joint. The least movement occurred at the cranial horn of

262 the medial meniscus [22]. The investigators speculated that this lack of movement induced

263 meniscal trapping between the femur and tibia in hyperextension that could contribute to the high 
264 prevalence of lesions at this site. In contrast, in both man [28] and dogs [29], meniscal tears have

265 been predominantly diagnosed in the caudal horn. The cranial meniscal horns are more movable

266 than the posterior horns in man [29] and this may explain some of the species differences. The

267 forces on the caudal meniscal horn are also known to increase substantially throughout flexion of 268 both equine and human femorotibial joints [15; 23]. The increased incidence of caudal horn injuries

269 has been ascribed to this caudal translocation of load in man [15].

270 The change in equine meniscal conformation from a C-shape to an L-shape described by

271 Fowlie et al. [22] that arises during stifle flexion may place the meniscal inner border under tension

272 and could explain the fraying frequently observed at this location in all 3 meniscal segments in the

273 present study. Furthermore, Bonilla et al. [23] also reported that the center of the equine tibial

274 plateau, that has no meniscal tissue cover, sustained increased stress loads throughout stifle flexion

275 and could contribute to fraying of the meniscal inner border or formation of meniscal body tears.

Meniscal tears were also observed on the equine meniscal tibial surface in the present study and have not been described previously in horses. We speculate that this pathology may be related to the subchondral bone resorption we recently identified at the medial tibial plateau in equine

279 femorotibial OA joints [4]. These tears could potentially be visualized by ultrasound examination or MRI but would not be identified arthroscopically.

The presence of osteophytes is considered pathognomic for the presence of $\mathrm{OA}$ in equine 282 joints [30]. Both the macroscopic and histologic meniscal scores were positively associated with

283 the presence of osteophytes in the medial femorotibial joint underpinning a likely association

284 between both events in this joint. It is well known in man $[15 ; 31]$ and has recently been shown in

285 horses that the medial femorotibial compartment is the most commonly affected by OA in the stifle

286 joint [4]. It is also increasingly recognized that meniscal injuries contribute to femorotibial OA 287 [17], though it has never been studied in horses. A large percentage (44\%) of patients with meniscal 
tears diagnosed on arthroscopic examination had accompanying cartilage lesions [31] Meniscal

289 degeneration also increases with OA severity in man $[17 ; 18]$. The correlation we observed between

290 equine meniscal degeneration and OA does not imply causation and further studies will be required

291 to establish where the earliest changes arise: in the meniscus or the articular cartilage or both

292 concurrently. As both the meniscus and articular cartilage are tightly interlinked anatomically and

293 biomechanically, loss of biomechanical function of either tissue through a single event trauma or

294 as a result cyclical stress induced injury will impact the other. Similarly, biological events such as

295 cellular activation of the pro-inflammatory/protease cascades in either tissue or the joint may

296 upregulate degradation of their extracellular matrices. A recent 3 year longitudinal study,

297 employing quantitative MRI (3T), imaged human patients with posterior meniscal horn lesions but

298 no radiographic OA or MRI cartilage lesions at study entry [32]. The investigators detected

299 elevated cartilage relaxation times, reflecting matrix degeneration, adjacent to the meniscal lesions

300 at the medial tibial plateau at 2 years, but not in matched controls. This finding supports the

301 argument that meniscal lesions may contribute to, or be one of the first signs, of degenerating

302 cartilage. These recent findings suggest meniscal lesions contribute to the development of OA in

303 the femorotibial joints. However, in contrast Badlani et al. [33] also found no significant difference

304 between patients with or without medial meniscal tears and the development of OA, over a 2 year

305 period.

306 In the present study, there was a significant effect of age on meniscal degeneration scores.

307 Little is known about meniscal ageing in any species, but age is a known risk factor for the

308 development of OA in man [34]. In a study of the prevalence of meniscal damage in the general

309 population $(n=991)$ and the association of meniscal tears with knee symptoms and radiographic

310 OA, the prevalence of meniscal tear was as low at $19 \%$ in women $50-59$ years old and high as $56 \%$

311 in men from 70-90 years old. In people with radiographic OA, the prevalence of a meniscal tear 
312 was $63 \%$ in symptomatic and $60 \%$ in non-symptomatic patients. It was concluded that incidental

313 meniscal findings on MRI of the knee are common in the general population and augment with

314 increasing age [14]. These findings will need to be kept in mind as our capacity to image equine

315 menisci improves as it may be a challenge to determine whether all the lesions we detect are

316 actually symptomatic.

317 It is recognized that this study has some limitations. As many of the samples were obtained

318 from an abattoir, a complete history was not available and it was unknown if clinical signs were

319 associated with the lesions we report, except for 2 horses with a confirmed clinical diagnosis.

320 Additional numbers of specimens would have provided further insight on meniscal changes with

321 age. Moreover, it should be pointed out that meniscal tissue sectioning and slide preparation are

322 challenging, probably related to its very complex and resistant collagen structure and quality

323 histological sections for analysis are difficult to obtain. On the other hand, this is the first study to

324 report normal meniscal morphology and lesions with gold standard post mortem and histological

325 assessments. Future studies including more clinical specimens, with lameness localized to the

326 femorotibial joint by intraarticular anesthesia, and a variety of lesions, could shed additional light

327 on the clinical relevance of the findings we report here.

328 In summary, equine meniscal lesions were identified in all segments and on both articular

329 surfaces. Meniscal lesions are associated with OA in the medial femorotibial joint and increase

330 with age. The exact relationship between meniscal degeneration and femorotibial OA remains to

331 be elucidated. 
334 Table 1: Data on menisci included in the study

$335 \mathrm{Stb}=$ Standardbred, $\mathrm{QH}=$ Quarter horse, $\mathrm{WB}=$ Warmblood; $\mathrm{F}=$ Female, $\mathrm{G}=$ Gelding, $\mathrm{M}=$ Male;

$336 \mathrm{R}=$ Right, $\mathrm{L}=\mathrm{Left} ;$ Med $=$ Medial, $\mathrm{Lat}=$ Lateral; $\mathrm{ME}=$ macroscopic evaluation

$337 *$ Data from specimens 21, 22 and 23 were employed for distribution of lesions and meniscal 338 degradation only.

340 FIGURE LEGENDS

341

342 Fig 1: Study Design.

343 Cr: Cranial, B: Body, Ca: Caudal

345 Fig 2: Scores.

346 Macroscopic (a) and histologic (c) meniseal scores with examples (b, d). Arrowheads are pointing 347 at lesions.

349 Fig 3: Femoral surface meniscal lesions (Score 3).

350 a) Lesion in the caûdal horn of a lateral meniscus extending towards the body. b), c) \& d) Lesion 351 in anterior horn of the medial meniscus.

353 Fig 4: Meniscal lesion laterality and distribution. 
354 Comparison of the medial and lateral global macroscopic (a) and histologic meniscal (b) scores.

355 C) \& d) Global macroscopic scores in meniscal segments.

356

357 Fig 5: Correlation of meniscal pathological scores with OA lesions.

358

359 Fig 6: Association of meniscal degeneration with age

$360 \mathrm{P}$ values indicate that the global medial and lateral meniscal macroscopic and histologic scores

361 significantly increase with age. 


\section{REFERENCES}

[1] Singer, E.R., Barnes, J., Saxby, F. and Murray, J.K. (2008) Injuries in the event horse: training versus competition. Vet $J \mathbf{1 7 5}, \mathbf{7 6 - 8 1 .}$

[2] Jeffcott, L.B. and Kold, S.E. (1982) Stifle lameness in the horse: a survey of 86 referred cases. Equine Vet J 14, 31-39.

[3] Walmsley, J.R., Phillips, T.J. and Townsend, H.G. (2003) Meniscal tears in horses: an evaluation of clinical signs and arthroscopic treatment of 80 cases. Equine Vet J 35, 402406.

[4] De Lasalle, J., Alexander, K., Olive, J. and Laverty, S. (2016) COMPARISONS AMONG RADIOGRAPHY, ULTRASONOGRAPHY AND COMPUTED TOMOGRAPHY FOR EX VIVO CHARACTERIZATION OF STIFLE OSTEOARTHRITIS IN THE HORSE. Vet Radiol Ultrasound 57, 489-501.

[5] Peroni, J.F. and Stick, J.A. (2002) Evaluation of a cranial arthroscopic approach to the stifle joint for the treatment of femorotibial joint disease in horses: 23 cases (19981999). Journal of the American Veterinary Medical Association 220, 1046-1052.

[6] Walmsley, J.P. (2005) Diagnosis and treatment of ligamentous and meniscal injuries in the equine stifle. Vet Clin North Am Equine Pract 21, 651-672, vii.

[7] Adrian, A.M., Barrett, M.F., Werpy, N.M., Kawcak, C.E., Chapman, P.L. and Goodrich, L.R. (2015) A comparison of arthroscopy to ultrasonography for identification of pathology of the equine stifle. Equine Vet J.

[8] Cohen, J.M., Richardson, D.W., McKnight, A.L., Ross, M.W. and Boston, R.C. (2009) Longterm outcome in $\mathbf{4} 4$ horses with stifle lameness after arthroscopic exploration and debridement. Vet Surg 38, 543-551.

[9] Barrett, M.F., Frisbie, D.D., Mcllwraith, C.W. and Werpy, N.M. (2012) The arthroscopic and ultrasonographic boundaries of the equine femorotibial joints. Equine Vet $J 44,57-$ 63.

[10] Flynn, K.A. and Whitcomb, M.B. (2002) Equine meniscal injuries: a retrospective study of 14 horses. In: Proc AAEP. pp 249-254.

[11] De Busscher, V., Verwilghen, D., Bolen, G., Serteyn, D. and Busoni, V. (2006) Meniscal damage diagnosed by ultrasonography in horses: a retrospective study of 74 femorotibial joint ultrasonographic examinations (2000-2005). Journal of Equine Veterinary Science 26, 453-461. 
[12] Watts, A.E. and Nixon, A.J. (2006) Comparison of arthroscopic approaches and accessible anatomic structures during arthroscopy of the caudal pouches of equine femorotibial joints. Vet Surg 35, 219-226.

[13] Santos, M.P., Gutierrez-Nibeyro, S.D., McKnight, A.L. and Singh, K. (2014) GROSS AND HISTOPATHOLOGIC CORRELATION OF LOW-FIELD MAGNETIC RESONANCE IMAGING FINDINGS IN THE STIFLE OF ASYMPTOMATIC HORSES. Veterinary Radiology \& Ultrasound.

[14] Englund, M., Guermazi, A., Gale, D., Hunter, D.J., Aliabadi, P., Clancy, M. and Felson, D.T. (2008) Incidental meniscal findings on knee MRI in middle-aged and elderly persons. New England Journal of Medicine 359, 1108-1115.

[15] Walker, P.S., Arno, S., Bell, C., Salvadore, G., Borukhov, I. and Oh, C. (2015) Function of the medial meniscus in force transmission and stability. J Biomech 48,1383-1388.

[16] Binfield, P.M., Maffulli, N. and King, J.B. (1993) Patterns of meniscal tears associated with anterior cruciate ligament lesions in athletes. Injury 24, 557-561.

[17] Zarins, Z.A., Bolbos, R.I., Pialat, J.B., Link, T.M., Li, X., Souza, R.B. and Majumdar, S. (2010) Cartilage and meniscus assessment using T1rho and T2 measurements in healthy subjects and patients with osteoarthritis. Osteoarthritis Cartilage 18, 1408-1416.

[18] Arno, S., Bell, C.P., Xia, D., Regatte, R.R., Krasnokutsky, S., Samuels, J., Oh, C., Abramson, S. and Walker, P.S. (2016) Relationship between meniscal integrity and risk factors for cartilage degeneration. Knee 23, 686-691.

[19] Pauli, C., Grogan, S.P., Patil, S., Otsuki, S., Hasegawa, A., Koziol, J., Lotz, M.K. and D'Lima, D.D. (2011) Macroscopic and histopathologic analysis of human knee menisci in aging and osteoarthritis. Osteoarthritis Cartilage 19, 1132-1141.

[20] Siegel, S. and Castellan, N.J. (1988) Nonparametric statistics for the behavioral sciences, 2nd edn., McGraw-Hill, New York. pp xxiii, 399 p.

[21] Fowlie, J., Arnoczky, S., Lavagnino, M., Maerz, T. and Stick, J. (2011) Resection of Grade III cranial horn tears of the equine medial meniscus alter the contact forces on medial tibial condyle at full extension: an in-vitro cadaveric study. Vet Surg 40, 957-965.

[22] Fowlie, J.G., Arnoczky, S.P., Stick, J.A. and Pease, A.P. (2011) Meniscal translocation and deformation throughout the range of motion of the equine stifle joint: an in vitro cadaveric study. Equine Vet J 43, 259-264. 
[23] Bonilla, A.G., Williams, J.M., Litsky, A.S. and Santschi, E.M. (2015) Ex Vivo Equine Medial Tibial Plateau Contact Pressure With an Intact Medial Femoral Condyle, With a Medial Femoral Condylar Defect, and After Placement of a Transcondylar Screw Through the Condylar Defect. Veterinary Surgery 44, 289-296.

[24] Fowlie, J.G., Arnoczky, S.P., Lavagnino, M. and Stick, J.A. (2012) Stifle extension results in differential tensile forces developing between abaxial and axial components of the cranial meniscotibial ligament of the equine medial meniscus: a mechanistic explanation for meniscal tear patterns. Equine Vet J 44, 554-558.

[25] Fregly, B.J., Besier, T.F., Lloyd, D.G., Delp, S.L., Banks, S.A., Pandy, M.G. and D'Lima, D.D. (2012) Grand challenge competition to predict in vivo knee loads. J Orthop Res 30, 503513.

[26] Halder, A., Kutzner, I., Graichen, F., Heinlein, B., Beier, A. and Bergmann, G. (2012) Influence of limb alignment on mediolateral loading in total knee replacement. The Journal of Bone \& Joint Surgery 94, 1023-1029.

[27] Griffin, T.M., Main, R.P. and Farley, C.T. (2004) Biomechanics of quadrupedal walking: how do four-legged animals achieve inverted pendulum-like movements? J Exp Biol 207, 3545-3558.

[28] Thompson, W.O., Thaete, F.L., Fu, F.H. and Dye, S.F. (1991) Tibial meniscal dynamics using three-dimensional reconstruction of magnetic resonance images. Am J Sports Med 19, 210-215; discussion 215-216.

[29] Bennett, D. and May, C. (1991) Meniscal damage associated with cruciate disease in the dog. Journal of small animal practice 32, 111-117.

[30] Olive, J., d'Anjou, M.A., Alexander, K., Beauchamp, G. and Theoret, C.L. (2010) Correlation of signal attenuation-based quantitative magnetic resonance imaging with quantitative computed tomographic measurements of subchondral bone mineral density in metacarpophalangeal joints of horses. Am J Vet Res 71, 412-420.

[31] Christoforakis, J., Pradhan, R., Sanchez-Ballester, J., Hunt, N. and Strachan, R.K. (2005) Is there an association between articular cartilage changes and degenerative meniscus tears? Arthroscopy 21, 1366-1369.

[32] Russell, C., Pedoia, V., Souza, R.B. and Majumdar, S. (2016) Cross-sectional and longitudinal study of the impact of posterior meniscus horn lesions on adjacent cartilage composition, patient-reported outcomes and gait biomechanics in subjects without radiographic osteoarthritis. Osteoarthritis Cartilage. 
491

492

493

494

495

496

497

498
[33] Badlani, J.T., Borrero, C., Golla, S., Harner, C.D. and Irrgang, J.J. (2013) The effects of meniscus injury on the development of knee osteoarthritis: data from the osteoarthritis initiative. Am J Sports Med 41, 1238-1244.

[34] Loeser, R.F. (2009) Aging and osteoarthritis: the role of chondrocyte senescence and aging changes in the cartilage matrix. Osteoarthritis Cartilage 17, 971-979.

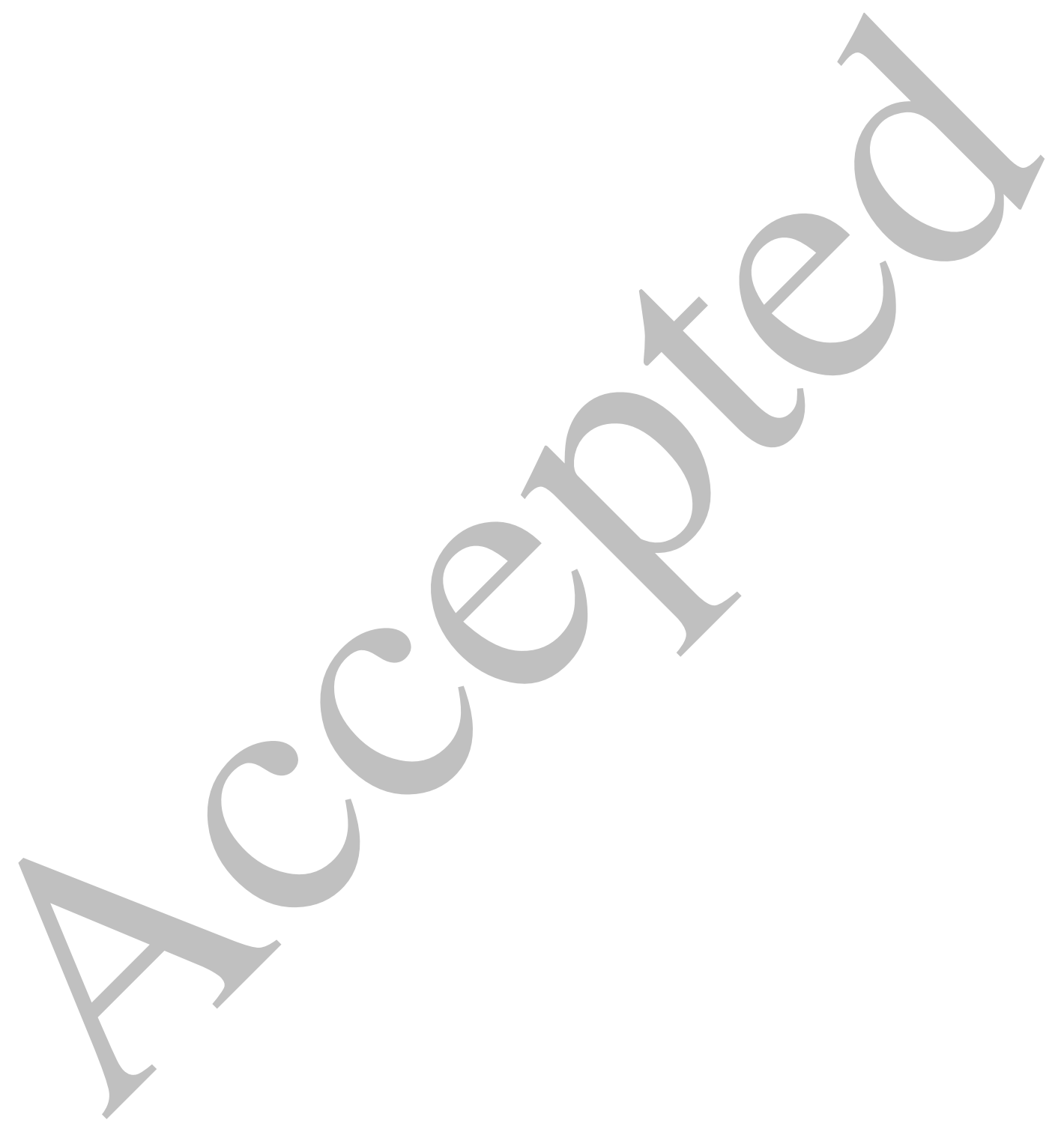


